\title{
Abundance of denitrifying genes and microbial community structure in volcanic soils
}

\author{
A.M. Carvajal*, R.A. Vargas, M. Alfaro
}

Instituto de Investigaciones Agropecuarias, Laboratorio de Biotecnología. INIA Remehue, Carretera Panamericana Sur km 8 Norte, Osorno, Chile. *Corresponding author: andres.carvajal@inia.cl

\begin{abstract}
Nitrous oxide $\left(\mathrm{N}_{2} \mathrm{O}\right)$ is a potent greenhouse gas produced during denitrification, a process that includes several genes coding enzymes responsible for nitrogen $(\mathrm{N})$ forms transformations. In volcanic ash soils of southern Chile, fertilization had increased over the last 10 years with implications for $\mathrm{N}_{2} \mathrm{O}$ emissions. Despite this, little is still known about potential denitrification and the presence of denitrifying genes. In this study we report the abundance of denitrifying genes and a basal characterization of microbial communities in five volcanic ash soils with different levels of organic matter. The denitrifying genes determination showed that nosZI predominated over nirK and nir $\mathrm{S}$ in all soils suggesting a complete denitrification pathway, which would explain low $\mathrm{N}_{2} \mathrm{O}$ losses in such soils. Estimation of total microorganisms studies using $16 \mathrm{~S}$ and $18 \mathrm{~S}$ rRNA showed that in these soils bacteria $\left(10^{9}\right)$ was most abundant over archaea and fungi $\left(10^{7} ; \mathrm{p}<0.05\right)$. Sequences of DNA obtained by denaturant gradient gel electrophoresis (DGGE) indicated that Firmicutes, Acidobacteria and Proteobacteria are the main bacterial classes represented in Andisoils (33, 28 and 23\%, respectively) but with a lower diversity. More detailed studies about denitrifiers, gene-activity relationship and $\mathrm{N}_{2} \mathrm{O}$ emissions are required.
\end{abstract}

Keywords :Volcanic soils, denitrification, nitrous oxide, denitrifying genes, microbial diversity 


\section{Introduction}

Agricultural soils are the main source of nitrous oxide $\left(\mathrm{N}_{2} \mathrm{O}\right)$ worldwide. Almost 300 times more influential that carbon dioxide $\left(\mathrm{CO}_{2}\right), \mathrm{N}_{2} \mathrm{O}$ contributes $10 \%$ to climate change each year and it is involved in the loss of the ozone layer (Thompson et al., 2012). This gas is produced in the nitrogen $(\mathrm{N})$ cycle, one of the major biogeochemical processes that is taking place in the biosphere. The cycle consists of two steps, nitrification and denitrification. The latter is an anaerobic respiration process involving a sequential and modular reduction of $\mathrm{N}$ species from nitrate $\left(\mathrm{NO}_{3}^{-}\right)$or nitrite $\left(\mathrm{NO}_{2}^{-}\right)$to nitric oxide $(\mathrm{NO})$, nitrous oxide $\left(\mathrm{N}_{2} \mathrm{O}\right)$ and nitrogen gas $\left(\mathrm{N}_{2}\right)$. Each one of these reductive steps is catalyzed by enzyme complexes: Nar, Nir, Nor and $\mathrm{N}_{2}$ or, respectively, which in turn are encoded by $n a r \mathrm{G}$ and napA genes for Nar, nirK and nirS for Nir, nor $\mathrm{B}$ and qnor $\mathrm{B}$ for Nor, and nos $\mathrm{Z}$ for the complex $\mathrm{N}_{2}$ or (Philippot et al., 2007). The description of this process has been mainly described in different bacterial communities, but its role in Archaea and Fungi domains it has only been recently studied (Maeda et al., 2015).

In Chile, agriculture is mainly carried out in the central and southern region of the country on volcanic ash soils (Andisoils, Alfisoils and Ultisoils) with a varied pattern of physicochemical properties including soil organic matter (SOM) content (Escudey et al., 2001). In Andisoils, Alfaro and Salazar (2008) have reported higher $\mathrm{N}$ losses as $\mathrm{NO}_{3}$ whereas Vistoso et al. (2012) showed lower $\mathrm{N}_{2} \mathrm{O}$ emissions after $\mathrm{N}$ application, suggesting that denitrification and $\mathrm{N}_{2} \mathrm{O}$ emissions are limited and likely to be carried out completely, favoring $\mathrm{N}_{2}$ formation. A preliminary study of the abundance of genes linked to the denitrification process showed that in two Andisoils with high SOM, the presence of bacterial nos $\mathrm{Z}$ gene was abundant, supporting the idea that in these soils denitrification was carried out comprehensively (Cardenas et al., 2013). However, no information is available in relation to denitrifying genes in others soil types, neither the abundance and microbial composition related to denitrification in other domains.

The objectives of this study were i) to quantify the abundance of bacterial denitrifying genes (nir $\mathrm{K}, n i r \mathrm{~S}$ and nosZI) in volcanic ash soils, and ii) to evaluate the bacterial diversity and its relative abundance to archaea and fungi domains in these soils.

\section{Materials and Methods}

\subsection{Experimental sites and soil sampling}

Five soil series were selected providing a range of SOM content: Collinco (36 $43^{\circ}$ 'S, $\left.71^{\circ} 54^{\prime} \mathrm{W}\right)$, Arrayán

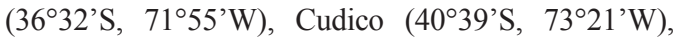
Osorno (40³1'S, $\left.73^{\circ} 02^{\prime} \mathrm{W}\right)$ and Nueva Braunau $\left(41^{\circ} 25^{\prime} \mathrm{S}, 73^{\circ} 24^{\prime} \mathrm{W}\right)$, with SOM contents of $4,16,15$, 21 and $45 \%$, respectively (Table 1 ). Soil samples ( 0 to $10 \mathrm{~cm}$ depth, $\mathrm{n}=3$ ) were taken between august 2013 and march 2014 from permanent grassland sites with no recent history of $\mathrm{N}$ fertilizer application or grazing, and transported to INIA Remehue ( $\left.40^{\circ} 35^{\prime} \mathrm{S}, 73^{\circ} 12^{\prime} \mathrm{W}\right)$, where they were thoroughly mixed, sieved to $2 \mathrm{~mm}$ and stored at $-80^{\circ} \mathrm{C}$. The physicochemical analysis of soils (Table 1) was performed according to Sadzawka et al. (2006) and Lobos Ortega et al. (2016).

\subsection{DNA extraction}

Total DNA was extracted from $0.3 \mathrm{~g}$ of soil from each series using the PowerSoil ${ }^{\mathrm{TM}}$ DNA Isolation Kit (MO BIO Laboratories, Inc., USA) and according to the manufacturer's instruction. The DNA was quantified by spectrophotometry using an Infinite M200 Nano- 
Quant microplate reader (Tecan Trading AG; Switzerland) and its quality was evaluated by agarose gel electrophoresis in TAE 1x buffer stained with GelRed
(Biotium, USA) as mentioned in Armas-Ricard et al (2016). The DNA samples were stored in aliquots at $-80{ }^{\circ} \mathrm{C}$ until amplification.

Table 1. Main physicochemical soil characteristics $(0-10 \mathrm{~cm}$ depth; $\mathrm{n}=3)$.

\begin{tabular}{|c|c|c|c|c|c|}
\hline Parameter & Collinco & Arrayán & Cudico & Osorno & Nueva Braunau \\
\hline Sampling date & December'13 & December'13 & September'13 & August'13 & March'14 \\
\hline Soil type & Alfisol & Andisol & Ultisol & Andisol & Andisol \\
\hline \multirow[t]{2}{*}{ Classification $^{1}$} & Typic & Typic & Typic & Typic & Typic \\
\hline & Rhodoxeralfs & Melanoxerands & Hapludults & Hapludands & Hapludands \\
\hline Soil texture & Sandy clay & Sandy loam & Clay & Clay loam & Loam \\
\hline SOM $(\%)$ & $4.0 \pm 0.06^{\mathrm{d}}$ & $14.9 \pm 0.28^{\mathrm{c}}$ & $16.4 \pm 0.48^{\mathrm{c}}$ & $21.4 \pm 0.55^{b}$ & $44.1 \pm 1.20^{\mathrm{a}}$ \\
\hline $\mathrm{C} / \mathrm{N}$ & $10^{\mathrm{c}}$ & $13^{b}$ & $13^{b}$ & $14^{b}$ & $17^{\mathrm{a}}$ \\
\hline $\mathrm{N}\left(\mathrm{mg} \mathrm{kg}^{-1}\right)$ & $4.5 \pm 0.50^{\mathrm{c}}$ & $8.0 \pm 0.01^{b c}$ & $7.5 \pm 0.29^{c}$ & $67.8 \pm 2.59^{a}$ & $13.0 \pm 0.71^{b}$ \\
\hline P Olsen $\left(\mathrm{mg} \mathrm{kg}^{-1}\right)$ & $2.5 \pm 0.06^{\mathrm{e}}$ & $15.7 \pm 0.20^{\mathrm{d}}$ & $23.3 \pm 0.88^{\mathrm{c}}$ & $51.5 \pm 0.67^{\mathrm{a}}$ & $36.0 \pm 1.28^{b}$ \\
\hline $\mathrm{Al}$ sat $(\%)$ & $22.4 \pm 0.18^{a}$ & $0.28 \pm 0.021^{\mathrm{d}}$ & $12.5 \pm 0.28^{b}$ & $0.72 \pm 0.03^{d}$ & $1.58 \pm 0.565^{\mathrm{c}}$ \\
\hline $\mathrm{Cu}\left(\mathrm{mg} \mathrm{kg}^{-1}\right)$ & $1.98 \pm 0.011^{\mathrm{c}}$ & $2.28 \pm 0.020^{\mathrm{c}}$ & $5.74 \pm 0.123^{a}$ & $5.37 \pm 0.128^{a}$ & $4.33 \pm 0.258^{b}$ \\
\hline $\mathrm{pH}\left(\mathrm{H}_{2} \mathrm{O}\right)$ & $5.37 \pm 0.015^{\mathrm{e}}$ & $6.22 \pm 0.006^{\mathrm{a}}$ & $5.52 \pm 0.003^{\mathrm{d}}$ & $5.80 \pm 0.014^{b}$ & $5.70 \pm 0.008^{\mathrm{c}}$ \\
\hline
\end{tabular}

1, Classification according to CIREN (2003); SOM: soil organic matter; C/N: carbon to nitrogen ratio; N, nitrogen; $\mathrm{P}$, phosphorus; $\mathrm{Al}$ sat, aluminum saturation; $\mathrm{Cu}, \mathrm{Cu}^{2+}$ concentration.

\subsection{Quantitative PCR}

Amplification of gene fragments was performed on a Rotor-Gene 6000 (Qiagen, Germany) using published primers (Table 2). Three replicates of each soil were used and run in triplicate. All amplifications were performed using the SensiFAST HRM Kit (Bioline Reagents Ltd.; UK), each reaction containing a final concentration of $1 \mathrm{X}$ SensiFAST HRM Mix, $400 \mathrm{nM}$ Forward primer, $400 \mathrm{nM}$ Reverse primer, $10 \mathrm{ng}$ of DNA template and DNase-free water in
$20 \mathrm{uL}$ of reaction. Each amplification included a negative control without DNA (NTC).

For denitrifying genes, the copy number was normalized to account for soil moisture and to bacterial $16 \mathrm{~S}$ rRNA to standardize by the total number of bacteria in the sample. The final genes copy numbers were reported as per gram of dry soil using the bulk density of the soil as measured at the collection site at the sampling time (Rowell, 1997). The potential transformation of $\mathrm{N}_{2} \mathrm{O}$ to $\mathrm{N}_{2}$ was estimated as nos ZI/ (nir $\mathrm{K}+$ nir $\mathrm{S})$ ratio. 
Table 2. Genes, primers and conditions used in the microbial determinations.

\begin{tabular}{|c|c|c|c|c|c|}
\hline Gen & Primer & Sequence $5^{\prime}-3^{\prime}$ & $\begin{array}{c}\mathrm{T} \text { annealing } \\
\left({ }^{\circ} \mathrm{C}\right)\end{array}$ & Amplicon & Reference \\
\hline 16S rRNA & R907* & CCGTCAATTCMTTTGAGTTT & 58 & 549 bp & Muyzer et al., \\
\hline Bacteria & F358 & CCTACGGGAGGCAGCAG & & & 1993. \\
\hline 16S rRNA & UA1406R & ACG GGC GGT GWG TRC AA & 55 & 655 bp & Baker, et al., \\
\hline Archea & A751F & CCG ACG GTG AGR GRY GAA & & & 2003. \\
\hline $18 \mathrm{~S}$ rRNA & FR1 & AIC CAT TCA ATC GGT AIT & 53 & $390 \mathrm{bp}$ & Vainio and \\
\hline Fungi & FF390 & CGA TAA CGA ACG AGA CCT & & & Hantula, 2000. \\
\hline \multirow[t]{2}{*}{$\operatorname{nir} \mathrm{K}$} & nirK1F & GGMATGGTKCCSTGGCA & $63 * * / 58$ & $514 \mathrm{bp}$ & Braker, et al., \\
\hline & $\operatorname{nirK} 5 \mathrm{R}$ & GCCTCGATCAGRTTRTGG & & & 1998. \\
\hline \multirow[t]{2}{*}{$n i r \mathrm{~S}$} & $\mathrm{Cd} 3 \mathrm{aF}$ & GTSAACGTSAAGGARACSGG & 58 & $425 \mathrm{bp}$ & Michotey et al., \\
\hline & R3cd & GASTTCGGRTGSGTCTTGA & & & $2000 ; 2004$ \\
\hline \multirow[t]{2}{*}{$\operatorname{nos} \mathrm{ZI}$} & $\operatorname{nos} Z 2 F$ & CGC RAC GGC AAS AAG GTS MSS GT & 60 & $267 \mathrm{bp}$ & Henry et al., \\
\hline & nosZ2R & CAK RTG CAK SGC RTG GCA GAA & & & 2006. \\
\hline
\end{tabular}

*The G-C Clamp sequence

CGCCCGCCGCGCGCGGCGGGCGGGGCGGGGGCACGGGGGGC

CGTCAATTCMTTTGAGTTT, was attached to the $5^{\prime}$ end of the primer. ** Touchdown $\left(1^{\circ} \mathrm{C} /\right.$ cycle $)$.

\subsection{Calibration curves}

To discard detrimental effects of PCR inhibitors, calibration curves with several DNA dilutions were constructed for each gene under study and tested as in Cardenas et al. (2013). The amplicons of each gene were cloned in E. coli JM109 using pGEM ${ }^{\circledR}$-T Easy Cloning Vector (Promega, USA) according to the manufacturer's instructions. Plasmid DNA was purified using the PureYield ${ }^{\mathrm{TM}}$ Plasmid Miniprep System (Promega, USA) and its concentration was determined by spectrophotometry, as above. The presence of the inserts was verified by PCR with their specific primers and subsequent gel electrophoresis. The calibration curves since serial plasmid DNA showed a linear range between 106-1011 copy number, a $\mathrm{R}^{2}>0.99$ and the efficiency varied between $0.80-1.10$.

\subsection{Bacterial community analysis}

Denaturant Gradient Gel Electrophoresis (DGGE) analysis for bacterial 16S rRNA was performed using a Cipher DGGEK-2001 system (C.B.S. Scientific, USA) and a GC clamp sequence attached to the 5' end of the forward primer. Briefly, $17 \mu \mathrm{L}$ of PCR product was loaded onto an $8 \%(\mathrm{w} / \mathrm{v})$ polyacrilamide gel with a $40-80 \%$ gradient. The electrophoresis was run for $17 \mathrm{~h}$ at $100 \mathrm{~V}$ and the gel was stained with GelRed (Biotium Inc., USA) for $30 \mathrm{~min}$ and photographed on a UV transilluminator (UVP, USA). Representative bands with a high reproducibility in DGGE gels were identified using the Doc-ItLS analysis software (UVP) and carefully excised with a scalpel to ensure the extraction of representative information about bacterial diversity. 
The DNA was purified by the crush and soak protocol (Sambrook and Rusell, 2001) and a second round of amplification was performed to ensure that excised bands did not contain multiple bands. Then, the amplicons were sequenced (Macrogen, Inc., Korea) and the chromatograms retrieved were compared to reported sequences at the National Center for Biotechnology Information (NCBI) and analyzed with BioEdit 7.2.5 and BLAST (http://blast.ncbi.nlm.nih-gov/Blast.cgi) programs. Taxonomic identification was performed with RDP Classifier (http://rdp.cme.msu.edu/classifier/classifier.jsp) and EzTaxon server (http://www.ezbiocloud.net/eztaxon/identify) databases. The total diversity was determined using the Shannon-Weaver index (Zar, 1999).

\subsection{Statistical analysis}

The gene copy number data were analyzed by a oneway ANOVA using the Statistica 7.0 package and comparisons were carried out with Fisher test. Differences were considered to be significant when the $p$ value was $\leq 0.05$. The global relationship between gene abundances and the physicochemical soil parameters was performed using a multivariate generalized Procrustes analyses (Info-Gen) according to Gower (1975), and the relationship between SOM concentration and gene abundances was estimated using a lineal regression test. In both cases, JMP 10.0 was used as statistical package.

Table 3. Abundance of genes studied in volcanic ash soils $(n=3)$. Data are showed as copy number $g$ soil ${ }^{-1} \pm$ standard error of the mean.

\begin{tabular}{|c|c|c|c|c|c|}
\hline & Collinco & Arrayán & Cudico & Osorno & Nueva Braunau \\
\hline nirK & $8.6 \times 10^{-4} \pm 9.82 \times 10^{-5} \mathrm{a}$ & $6.8 \times 10^{-4} \pm 1.93 \times 10^{-5} \mathrm{a}$ & $7.6 \times 10^{-4} \pm 8.79 \times 10^{-5} \mathrm{a}$ & $3.9 \times 10^{-4} \pm 2.58 \times 10^{-5 b}$ & $4.6 \times 10^{-4} \pm 2.44 \times 10^{-5 t}$ \\
\hline nirS & $2.5 \times 10^{-4} \pm 2.85 \times 10^{-5} \mathrm{bc}$ & $3.7 \times 10^{-4} \pm 5.31 \times 10^{-6 \mathrm{a}}$ & $3.1 \times 10^{-4} \pm 5.26 \times 10^{-5} \mathrm{ab}$ & $1.8 \times 10^{-4} \pm 1.01 \times 10^{-5 \mathrm{c}}$ & $2.7 \times 10^{-4} \pm 1.29 \times 10^{-5} \mathrm{t}$ \\
\hline $\operatorname{nos} \mathrm{ZI}$ & $4.6 \times 10^{0} \pm 9.13 \times 10^{-1} \mathrm{c}$ & $1.2 \times 10^{1} \pm 1.40 \times 10^{0 \mathrm{a}}$ & $5.5 \times 10^{0} \pm 5.23 \times 10^{-1} \mathrm{bc}$ & $8.3 \times 10^{0} \pm 9.08 \times 10^{-1 b}$ & $1.5 \times 10^{1} \pm 1.00 \times 10^{0 \mathrm{a}}$ \\
\hline nos $\mathrm{ZI} / \operatorname{nir} \mathrm{K}+$ nir $\mathrm{S}$ & $4.1 \times 10^{3} \pm 5.04 \times 10^{2} \mathrm{~d}$ & $1.1 \times 10^{4} \pm 1.35 \times 10^{3} \mathrm{c}$ & $5.5 \times 10^{3} \pm 6.87 \times 10^{2} \mathrm{~d}$ & $1.4 \times 10^{4} \pm 1.03 \times 10^{3 \mathrm{~b}}$ & $2.0 \times 10^{4} \pm 9.24 \times 10^{2 \mathrm{a}}$ \\
\hline $16 \mathrm{~S}$ rRNA Bacteria & $3.5 \times 10^{9} \pm 2.85 \times 10^{8} \mathrm{a}$ & $3.8 \times 10^{9} \pm 2.90 \times 10^{8} \mathrm{a}$ & $4.6 \times 10^{9} \pm 5.88 \times 10^{8} \mathrm{a}$ & $3.6 \times 10^{9} \pm 2.8 \times 10^{8} a$ & $4.1 \times 10^{9} \pm 3.43 \times 10^{8} \mathrm{a}$ \\
\hline $16 \mathrm{~S}$ rRNA Archaea & $5.8 \times 10^{7} \pm 3.21 \times 10^{5 b}$ & $6.3 \times 10^{7} \pm 2.31 \times 10^{5 a b}$ & $6.6 \times 10^{7} \pm 4.38 \times 10^{5} \mathrm{a}$ & $3.0 \times 10^{7} \pm 2.89 \times 10^{5} \mathrm{c}$ & $2.8 \times 10^{7} \pm 6.70 \times 10^{6 c}$ \\
\hline $18 \mathrm{~S}$ rRNA Fungi & $2.2 \times 10^{7} \pm 8.63 \times 10^{5 a}$ & $1.4 \times 10^{7} \pm 2.29 \times 10^{6 b}$ & $1.5 \times 10^{7} \pm 8.84 \times 10^{5 b}$ & $1.6 \times 10^{7} \pm 1.80 \times 10^{7 b}$ & $1.5 \times 10^{7} \pm 1.76 \times 10^{7 b}$ \\
\hline
\end{tabular}

Different letters indicate significant differences between soils $(\mathrm{p}<0.05)$. 


\section{Results}

\subsection{Quantification of denitrifying genes}

The quantification of denitrifying genes copy number showed that nosZI was the most abundant gene in all soils, followed by nirK and nirS ( $p<0.05$; Table 3$)$. For each gene, significant differences were found between soils. The abundance of nosZI was higher in andisoils (Nueva Braunau, Arrayán and Osorno) and lower in the ultisoil. For nirK the abundance was relatively inverse to nosZI with lower levels in Osorno and Nueva Braunau, and higher values in Collinco, Cudico and Arrayán soils $(p<0.05$; Table 3$)$. The abundance of nirS gene was higher in Arrayán and Cudico, and lower in Osorno, Collinco and Nueva Braunau soils. In addition, the nos ZI/nirK +nirS ratio was higher in andisoils ( $p<0.05$; Table 3 ).

The Procraster analyses to relate gene abundances and the total physicochemical soil parameters show a high percentage of consensus (94.1\%) whereas positive lineal relationships were found between SOM content and nosZI gene copy number $\left(\mathrm{R}^{2}=0.691\right)$ and the $\operatorname{nos} \mathrm{ZI} /$ nir $\mathrm{K}+$ nir $\mathrm{S}$ ratio $\left(\mathrm{R}^{2}=0.769\right)$.

\subsection{Abundance and diversity of microorganisms}

The abundance of the bacterial 16S rRNA gene varied between $3.45 \times 10^{9}$ and $4.55 \times 10^{9}$ copy number $\mathrm{g}^{-1}$, without significant differences among soils $(p>0.05$; Table 3). For Archaea, 16S rRNA abundancy ranged between $2.83 \times 10^{7}$ and $6.64 \times 10^{7}$ and was significantly lower than in bacteria $(p<0.05)$.

Differences among soils were observed between the Osorno and Nueva Braunau soil series containing lower abundancy whereas Cudico and Arrayán soils had higher contents (Table 3). Fungal 18S rRNA was lower than bacterial 16S rRNA and only showed a significant difference between Collinco and the others soils $(p<0.05)$.

The DGGE analysis for bacterial 16S rRNA thowed high number of bands $(>100)$, with different migration and intensities between soils (data not showed). We obtained a total of 57 DNA sequences that were taxonomically classified to the Class level. Initially, nine sequences were inconsistent with those reported at NCBI but successfully assigned after RDP and/ or EzTaxon analysis. The taxonomic diversity in all analyzed soil series corresponded to nine phyla and 21 classes (Table 4). The most predominant phyla in whole soils were Firmicutes (33\%), Acidobacteria (28\%) and Proteobacteria (23\%). Firmicutes was predominant in Cudico (57\%), Osorno (46\%) and Nueva Braunau (50\%) soils, respectively, whereas Acidobacteria predominated in Collinco (58\%, Figure 1). Proteobacteria predominated in the Arrayán soil $(62 \%)$. Other phyla less represented were Actinobacteria, Bacteroidetes, Chloroflexi, Deinococcus-Thermus, Gemmatimonadetes and Calsiderica (Figure 1). The Shannon-Wiener index showed that the total diversity was low $\left(\mathrm{H}^{\prime}<1.0\right)$ with similar values between soils (Table 5). 
Table 4. Taxonomic identification of the DGGE bands.

\begin{tabular}{|c|c|c|c|c|c|c|}
\hline Soil & Band & Gen Reference NCBI & Identity $(\%)^{*}$ & Domain & Phylum & Class \\
\hline \multirow[t]{12}{*}{ Collinco } & 1 & Uncultured bactenium clone MRD120_B12 16S ribosomal RNA gene, partial sequence & 99 & Bacteria & Bacteroidetes & Sphingobacteria \\
\hline & 2 & Uncultured bacterium clone HL625 $16 \mathrm{~S}$ ribosomal RNA gene, partial sequence & 93 & Bacteria & Acidobacteria & Acidobacteria_Gp4 \\
\hline & 3 & Uncultured bacterium clone A1_a6 16 S ribosomal RNA gene, partial sequence & 91 & Bacteria & Acidobacteria & Acidobacteria \\
\hline & 4 & Uncultured bacterium partial $16 \mathrm{~S}$ rRNA gene, DGGE band FRV03-7 & 83 & Bacteria & Planctomycetes & Planctomycetacia \\
\hline & 5 & Uncultured bacterium clone FCPO $41016 \mathrm{~S}$ ribosomal RNA gene, complete sequence & 88 & Bacteria & Firmicutes & Clostridia \\
\hline & 6 & Uncultured bactenium clone B184 16 S ribos omal RNA gene, partial sequence & 77 & Bacteria & Proteobacteria & Gammaproteobacteria \\
\hline & 7 & Uncultured Acidobacteria bacterium clone SCGR $08216 \mathrm{~S}$ ribosomal RNA gene, partial sequence & 89 & Bacteria & Proteobacteria & Gammaproteobacteria \\
\hline & 8 & Uncultured bacterium clone B2 $16 \mathrm{~S}$ ribos omal RNA gene, partial sequence & 78 & Bacteria & Deinococcus-Thermus & Deinococci \\
\hline & 9 & Uncultured bacterium clone PAS1_B10 16S ribosomal RNA gene, partial sequence & 80 & Bactenia** & Acidobacteria ${ }^{* *}$ & Solibacteres** \\
\hline & 10 & $\begin{array}{l}16 \mathrm{~S} \text { rRNA amplicon fragment from a soil sample (ferralsol, Madagascar) resulting from a } 16 \text { days laboratory incubation } \\
\text { experiment in the presence of } 13 \mathrm{C} \text {-enriched wheat-straw: Heavy-DNA fraction (DNA-SIP technique) }\end{array}$ & 90 & Bacteria & Actinobacteria & Actinobacteria \\
\hline & 11 & $\begin{array}{l}16 \mathrm{~S} \text { rRNA amplicon fragment from a soil sample (ferralsol, Madagascar) resulting from a } 16 \text { days laboratory incubation } \\
\text { experiment in the presence of } 13 \mathrm{C}-\text {-enriched wheat-straw and a tropical peregrine endogeic earthworm, Pontoscolex } \\
\text { corethrurus: Light-DNA faction (DNASIP technique) }\end{array}$ & 88 & Bacteria & Actinobacteria & Actinobacteria \\
\hline & 12 & $\begin{array}{l}16 \mathrm{~S} \text { rRNA amplicon fragment from a soil sample (ferralsol, Madagascar) resulting from a } 16 \text { days laboratory incubation } \\
\text { experiment in the presence of } 13 \mathrm{C}-\text {-enriched wheat-straw: Light-DNA fraction (DNA-SIP technique) }\end{array}$ & 92 & Bacteria & Actinobacteria & Actinobacteria \\
\hline \multirow[t]{7}{*}{ Cudico } & 13 & Uncultured soil bacterium is olate DGGE gel band $1216 \mathrm{~S}$ ribosomal RNA gene, partial sequence & 77 & Bacteria** & Firmicutes** & Bacilli* $^{*}$ \\
\hline & 14 & Uncultured bacterium partial 16S rRNA gene, isolate MOTU101, clone MEDIUM2_BACSE6 & 92 & Bacteria & Firmicutes & Bacilli \\
\hline & 15 & Uncultured Fimicutes bacterium gene for $16 \mathrm{~S}$ nbosomal RNA, partial sequence, isolate: DGGE band P11_B18 & 76 & Bacteria & Firmicutes & Bacilli \\
\hline & 16 & Staphylococcus sp. Drenje $1.616 \mathrm{~S}$ nibosomal RNA gene, partial sequence & 98 & Bacteria & Firmicutes & Bacilli \\
\hline & 17 & Uncultured Terriglobus sp. clone M4011 $16 \mathrm{~S}$ rbosomal RNA gene, partial sequence & 80 & Bacteria* & Acidobacteria* ${ }^{*}$ & Acidobacteria_Gp1* \\
\hline & 18 & Uncultured soil bacterium is olate DGGE gel band $0216 \mathrm{~S}$ ribosomal RNA gene, partial sequence & $\$ 1$ & Bacteria & Firmicutes & Negativicutes \\
\hline & 19 & Uncultured bacterium partial $16 \mathrm{~S}$ rRNA gene, done $\mathrm{CuH} 21$ & 81 & Bacteria & Actinobacteria & Actinobacteria \\
\hline \multirow[t]{13}{*}{ Arrayán } & 20 & Uncultured Firmicutes bacterium partial 16S rRNA gene, clone EJR0S_21 & 86 & Bacteria & Firmicutes & Bacilli \\
\hline & 21 & Uncultured Acidobacteria bacterium clone SEG_08_135 16S nbosomal RNA gene, partial sequence & 82 & Bactenia** & Acidobacteria** & Acidobacteria_Gp4** \\
\hline & 22 & NCB unrated & & Bacteria & Bacteroidetes & Flavobacteria \\
\hline & 23 & Uncultured bactenum clone SII_2_47 16S ribosomal RNA gene, partial sequence & 77 & Bacteria & Firmicutes & Negativicutes \\
\hline & 24 & Uncultured bacterium clone $023016 \mathrm{~S}$ nibosomal RNA gene, partial sequence & 92 & Bacteria & Proteobacteria & Gammaproteobacteria \\
\hline & 25 & Uncultured bacterium clone HLUCs $35816 \mathrm{~S}$ ribosomal RNA gene, partial sequence & 90 & Bacteria & Firmicutes & Clostridia \\
\hline & 26 & Uncultured soil bacterium is olate DGGE gel band $1716 \mathrm{~S}$ ribosomal RNA gene, partial sequence & 74 & Bactenia** & Protobacteria** & Gammaprotobacteria** \\
\hline & 27 & Uncultured bacterium clone PS_B36016S nibosomal RNA gene, partial sequence & 96 & Bacteria & Proteobacteria & Gammaproteobacteria \\
\hline & 28 & Uncultured bactenum clone F3_E143 16S ribosomal RNA gene, partial sequence & 86 & Bacteria & Proteobacteria & Gammaproteobacteria \\
\hline & 29 & Bacterium endosymbiont of Onthophagus Taurus clone 3L2_NC1_019 16S ribosomal RNA gene, partial sequence & 97 & Bacteria & Proteobacteria & Gammaproteobacteria \\
\hline & 30 & Uncultured organism clone ELU0149-T297-S-NIPCRAMgANa_000468 small subunit nbosomal RNA gene, partial sequence & 73 & Bacteria & Chloroflexi & Thermomicrobia \\
\hline & 31 & Enterobacteriaceae bacterium M_Sw_oHS_07/11_1_1(1) $16 \mathrm{~S}$ ribosomal RNA gene, partial sequence & 97 & Bacteria & Proteobacteria & Gammaproteobacteria \\
\hline & 32 & Oceanisphaera sp. LAM-WHM-ZC $16 \mathrm{~S}$ ribosomal RNA gene, partial sequence & 76 & Bacteria & Proteobacteria & Betaproteobacteria \\
\hline \multirow[t]{13}{*}{ Osorno } & 33 & Uncultured alpha proteobacterium is olate DGGE gel band SPH-SW08-7 16S ribosomal RNA gene, partial sequence & 97 & Bacteria & Proteobacteria & Alphaproteobacteria \\
\hline & 34 & Uncultured bacterium clone SiteB3Y_207 16S ribosomal RNA gene, partial sequence & 90 & Bacteria & Fimicutes & Bacilli \\
\hline & 35 & Uncultured rumen bacterium clone MXAP1A-B1 16S ribosomal RNA gene, partial sequence & 97 & Bacteria & Firmicutes & Bacilli \\
\hline & 36 & Uncultured bacterium clone $17 \mathrm{~d}-2216 \mathrm{~S}$ ribosomal RNA gene, partial sequence & 91 & Bacteria & Fimicutes & Bacilli \\
\hline & 37 & Uncultured Fimicutes bacterium partial $16 \mathrm{~S}$ rRNA gene, clone DKBF_4 & 75 & Bacteria & Deinococcus-Thermus & Deinococci \\
\hline & 38 & Bacillus lentus $16 \mathrm{~S} r \mathrm{RNA}$ gene, is olate $\mathrm{P} 265 \mathrm{cl}$ & 96 & Bacteria & Fimicutes & Bacilli \\
\hline & 39 & Uncultured bacterium partial $16 \mathrm{~S}$ rRNA gene, done $\mathrm{DKBF}_{-} 5$ & 76 & Bacteria** & Protobacteria** & Alphaproteobacteria** \\
\hline & 40 & Uncultured bactenium clone BP_U4A_4g06 16S ribosomal RNA gene, partial sequence & 91 & Bacteria & Proteobacteria & Alphaproteobacteria \\
\hline & 41 & Uncultured bacterium gene for $16 \mathrm{~S}$ rRNA, partial sequence, done: $953 \mathrm{Sed} 68 \mathrm{u}$ & 82 & Bacteria & Chlorobi & Ignavibacteria \\
\hline & 42 & Uncultured bacterium clone D62 16S ribosomal RNA gene, partial sequence & 82 & Bacteria & Proteobacteria & Alphaproteobacteria \\
\hline & 43 & Uncultured soil bacterium isolate DGGE gel band $1116 \mathrm{~S}$ nibosomal RNA gene, partial sequence & 78 & Bacteria & Fimicutes & Clostridia \\
\hline & 44 & Uncultured bactenum RNA for $16 \mathrm{~S}$ rRNA, partial sequence, done: B1001R002_G23 & 92 & Bacteria & Firmicutes & Clostridia \\
\hline & 45 & Uncultured bactenium clone BP_U4B_2e10 16S ribosomal RNA gene, partial sequence & 79 & Bacteria & Planctomycetes & Planctomycetacia \\
\hline \multirow[t]{12}{*}{ S. Braunaul } & 46 & NCBI unrated & & Bacteria & Bacteroidetes & Sphingobacteria \\
\hline & 47 & Uncultured bacterium clone AFE_3_aao14h05 $16 \mathrm{~S}$ nibosomal RNA gene, partial sequence & 95 & Bacteria & Fimicutes & Bacilli \\
\hline & 48 & Uncultured bactenium gene for $16 \mathrm{~S} \mathrm{rRNA}$, partial sequence, done: $0502 \mathrm{TCLN} 172$ & 98 & Bacteria & Firmicutes & Bacilli \\
\hline & 49 & Bacillus sp. F3(2012) 16S ribosomal RNA gene, partial sequence & 77 & Bacteria** & Fimicutes** & Bacilli** \\
\hline & 50 & Uncultured Bacillus sp. isolate DGGE gel band $516 \mathrm{~S}$ nibosomal RNA gene, partial sequence & 100 & Bacteria & Firmicutes & Bacilli \\
\hline & 51 & Uncultured soil bacterium is olate DGGE gel band $1216 \mathrm{~S}$ ribosomal RNA gene, partial sequence & 76 & Bacteria & Cyanobacteria / Chloroplast & Chloroplast \\
\hline & 52 & Uncultured Burkholderia sp. isolate DGGE gel band 9TAT 16S ribosomal RNA gene, partial sequence & 76 & Bacteria & Proteobacteria & Deltaproteobacteria \\
\hline & 53 & Uncultured soil bacterium isolate DGGE gel band 1216 S ribosomal RNA gene, partial sequence & 74 & Bacteria & Firmicutes & Clostridia \\
\hline & 54 & Uncultured bacterium clone GB4XUSJOSJVLQW $16 \mathrm{~S}$ ribosomal RNA gene, partial sequence & 95 & Bacteria & Acidobacteria & Acidobacteria_Gp1 \\
\hline & 55 & Uncultured bacterium RNA for $16 \mathrm{~S}$ rRNA, partial sequence, done: B1001R002_G23 & 96 & Bacteria & Acidobacteria & Acidobacteria_Gp1 \\
\hline & 56 & Uncultured bacterium clone $16 \mathrm{~S}-\mathrm{T} 6-3-9 \mathrm{H} 16 \mathrm{~S}$ nbosomal RNA gene, partial sequence & 98 & Bacteria & Acidobacteria & Acidobacteria_Gp1 \\
\hline & 57 & Uncultured bacterium clone Upland_500_4065 16 S ribosomal RNA gene, partial sequence & 93 & Bacteria & Gemmatimonadetes & Gemmatimonadales \\
\hline
\end{tabular}

*Sequences assigned using NCBI; **Sequences assigned using the EzTaxon database. 


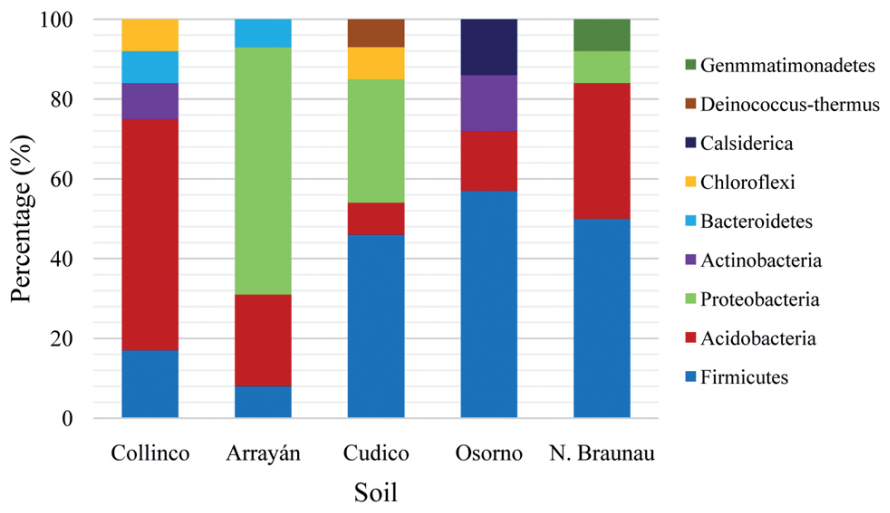

Figure 1. Phyla distribution of bacterial $16 \mathrm{~S}$ rRNA in volcanic ash soils.

Table 5. Diversity of bacterial phyla of volcanic ash soils measured as Shannon-Wiener index. H', diversity index; H' max, maximum diversity index; E, evenness.

\begin{tabular}{lccc}
\hline Soil & $\mathrm{H}^{\prime}$ & $\mathrm{H}^{\prime} \max$ & $\mathrm{E}$ \\
\hline Collinco & 0,6805 & 0,6989 & 0,9736 \\
Arrayan & 0,4480 & 0,6020 & 0,7466 \\
Cudico & 0,5010 & 0,6020 & 0,8322 \\
Osorno & 0,5695 & 0,6989 & 0,8148 \\
Nueva Braunau & 0,4894 & 0,6020 & 0,8129 \\
\hline
\end{tabular}

\section{Discussion}

In this study we performed qPCR to determine the abundance of denitrifying genes in different soil series currently used in agricultural or livestock activities in southern Chile. Our findings showed that nosZI gene was more abundant that nirK and $n i r \mathrm{~S}$ in all soils with similar values to several reports from around the world (Henry et al., 2006; Chronakova et al., 2009; Cardenas et al., 2013). In addition, andisoils showed a higher nos $\mathrm{ZI} /$ nir $\mathrm{K}+$ nir S ratio com- pared to alfisoil and ultisoil. According to this, a high relationship was found between nosZI or the potential transformation of $\mathrm{N}_{2} \mathrm{O}$ to $\mathrm{N}_{2}$ (nos ZI/nirK+nirS ratio) and SOM. These results suggest that during denitrification, most $\mathrm{NO}$ and $\mathrm{N}_{2} \mathrm{O}$ are reduced to $\mathrm{N}_{2}$ resulting in lower $\mathrm{N}_{2} \mathrm{O}$ emissions. This hypothesis is supported by the fact that denitrification have been defined as a poor-represented process in Chilean andisoils with lower $\mathrm{N}_{2} \mathrm{O}$ emissions compared to others reports (Vistoso et al., 2012). 
However, it has been reported that levels of $\mathrm{N}_{2} \mathrm{O}$ emissions could be uncoupled from genes abundance (Čuhel et al., 2010), so our results must be analyzed on a wider context. Furthermore, the study of transcripts or the real enzyme activities remain as significant areas to be investigated.

Denitrifiers had been traditionally classified into two functional and mutually exclusive groups, those carrying nirK or nirS genes, which uses $\mathrm{Cu}^{2+}$ and cytochrome $c d 1$, respectively, as co-factors (Philippot et al., 2007). But recently the co-occurrence of both genes in microbial communities have been reported (Graf et al., 2014). Regarding $\mathrm{N}_{2} \mathrm{O}$ reductase, recent phylogeny studies have reported another gene coined nosZII which would have a significant contribution to $\mathrm{N}_{2} \mathrm{O}$ losses, being associated to a different clade (Clade II; Jones et al., 2012). This nosZII gene is very widespread and diverse, and more abundant than nosZI in bacteria and archaea. Preliminary results of our group show that bacterial nosZII is present in our soils and future studies are necessary to highlight and clarify the importance and function of this gene in volcanic ash soils. Recent reports indicate that both Archaea and Fungi have homologous and analogous enzymes to carry out the process of denitrification (Maeda et al., 2015; Rusch, 2013), becoming a component to consider in future studies to cover the total contribution of microorganisms.

Chilean volcanic ash soils have been characterized in a limited way in terms of microbiota and its relation to denitrifiers. Our results using $16 \mathrm{~S}$ and $18 \mathrm{~S}$ rRNA indicates that Bacteria domain predominated in all evaluated soils, whereas Archaea is higher than Fungi. The 16S rRNA have been the universal marker used to obtain and estimate total abundance of bacteria and archaea in different samples (Prévost-Bouré et al., 2014) including rhizosphere and bulk soil (Lagos et al. 2015). Despite being a multicopy gene in several species, its quantification is similar to rpoB, a new sin- gle copy marker, validating their use in environmental samples (Deslippe et al., 2014). In our study the copy number of bacterial $16 \mathrm{~S}$ rRNA ranged between $3.45 \mathrm{x}$ $10^{8}$ to $4.55 \times 10^{8}$ being similar to another reports with soils showing a high SOM content (Kandeler et al. 2006; Cardenas et al. 2013). We found no significant differences between soils, despite the different physicochemical characteristics including SOM, pH or $\mathrm{C} / \mathrm{N}$ ratio. These results suggest that in volcanic soils, the abundance of microorganisms could be linked to others factors like $\mathrm{pH}$, water and oxygen content, organic $\mathrm{C}$ or the state of bioavailability of organic matter, regulating soil quality and plant nutrition (Čuhel et al., 2010; Barea, 2015). A similar trend was found for fungal 18S rRNA but with Collinco soil showing a higher abundance. For archaeal 16S rRNA, the pattern was more heterogeneous with andisoils showing the lower abundance. The gene copy number values reported here were similar to those reported by Cardenas et al. (2013) on similar soil types.

To assess the snapshot of the bacterial communities in our soils we performed DGGE assays, a molecular technique that is independent of culture and allows to study the diversity of communities (Muyzer et al, 1993). The pattern of bands obtained was different for each soil suggesting a differential composition. The sequence analysis only retrieved information to the Class level, and the total diversity measured as the Shannon-Weaver index was reduced probably because of the high taxonomic rank used in the allocation. This was presumably associated to the low number of DGGE bands isolated, which is recognized as the main limitation of the method. It is estimated that a single soil sample can generate a high number of bands (over 100 and more) corresponding to different communities (Nakatsu et al., 2007). Nonetheless, the information available allowed for the analysis of differences in the most represented phyla. Thus, the phyla with greater representation in the analyzed soils 
corresponded to Firmicutes, Acidobacteria and Proteobacteria, in agreement with Jorquera et al. (2010) in andisoil. While these phyla are considered significant for the denitrification process, specific phylogenetic studies are required for denitrifying genes because they are uncoupled of the proposed 16S rRNA evolution (Goregues et al., 2005). At the moment, we are performing DGGE using specific nosZI and nosZII primers.

Our results suggest than in volcanic soils of southern Chile, total denitrification can be expected under natural conditions which would explain the low $\mathrm{N}_{2} \mathrm{O}$ emissions registered for agricultural and grassland soils (Muñoz et al., 2011; Vistoso et al., 2012; Hube et al., 2016). Nevertheless, more studies including gene expression, enzyme activity and community structure of denitrifiers are required to link potential denitrification and the effect of soil macronutrients over $\mathrm{N}_{2} \mathrm{O}$ emissions. This knowledge will be also relevant to develop future mitigation strategies appropriate for specific soil characteristics.

\section{Conclusions}

Our results show that in our volcanic ash soils bacterial nosZI predominates between denitrifying genes. The high ratio of nosZI over nirK and nirS in Andisoils could suggest a higher denitrification potential. Compared to archaea and fungi, bacteria domain was most represented and includes Firmicutes, Acidobacteria and Proteobacteria classes, which have been implicated in soil denitrification. Further studies are necessary to understand soil $\mathrm{N}_{2} \mathrm{O}$ emissions and its mitigation.

\section{Acknowledgments}

This work was funded by the Fondo Nacional de Desarrollo Científico y Tecnológico (FONDECYT regular grant 1130718). We thank to Luis Ramírez and Sara Hube (INIA Remehue) for their assistance in soil sampling and physicochemical characterization, respectively, and Horacio Miranda for their assistance in statistical methods.

\section{References}

Alfaro, M., Salazar, F. 2008. Livestock production and diffuse pollution in a volcanic soil. J. Soil Sci. Plant Nutr. 8, 1-8.

Armas-Ricard, M., Orlando, J., Bustamante, R., Carú, M. 2016. Microbial communities of bulk and Eschscholzia californica rhizosphere soils at two altitudes in Central Chile. J. Soil Sci. Plant Nutr. $16,1-13$

Baker, G. C., Smith, J. J., Cowan, D.A. 2003. Review and re-analysis of domain-specific $16 \mathrm{~S}$ primers. J. Microbiol Meth. 55, 541-555.

Barea, J. M. 2015. Future challenges and perspectives for applying microbial biotechnology in sustainable agriculture based on a better understanding of plant-microbiome interactions. J. Soil Sci. Plant Nutr. 15, 261-282.

Braker, G., Fesefeldt, A., Witzel, K. 1998. Development of PCR primer systems for amplification of nitrite reductase genes (nirK and nirS) to detect denitrifying bacteria in environmental samples. Appl. Environ. Microbiol. 64, 3769-3775.

Cardenas, L. M., Hatch, D. J., Scholefield, S., Jhurreea, D., Clark, L.M., Hirsch, P.R., Salazar, F., Rao-Ravella, S., Alfaro, M. 2013. Potential mineralization and nitrification in volcanic grassland soils in Chile. J. Soil Sci. Plant Nutr. 59, 1-12.

Chroňáková, A., Radl, V. 2009. Overwintering management on upland pasture causes shifts in an abundance of denitrifying microbial communities, their activity and $\mathrm{N}_{2} \mathrm{O}$ reducing ability. Soil Biol. Biochem. 41, 1132-1138. 
CIREN. 2003. Descripciones de suelos, materiales y símbolos. Estudio Agrológico X Región. Vol. II. Centro de Información de Recursos Naturales (CIREN), Santiago.

Čuhel, J., Šimek, M., Laughlin, R. J., Bru, D., Chèneby, D., Watson, C.J., Philippot, L. 2010. Insights into the effect of soil $\mathrm{pH}$ on $\mathrm{N}_{2} \mathrm{O}$ and $\mathrm{N}_{2}$ emissions and denitrifier community size and activity. Appl. Environ. Microbiol. 76, 1870-1878.

Deslippe, J. R., Jamali, H., Jha, N., Saggar, S. 2014. Denitrifier community size, structure and activity along a gradient of pasture to riparian soils. Soil Biol. Biochem. 71, 48-60.

Escudey, M., Galindo, G., Förster, J. E., Briceño, M., Díaz, P., Chang, A. 2001. Chemical forms of phosphorus of volcanic ash-derived soils in Chile. Commun. Soil Sci. Plant Anal. 32, 601-616.

Goregues, C., M., Michotey, V., D., Bonin, P. 2005. Molecular, biochemical and physiological approaches for understanding the ecology of denitrification. Microbiol. Ecol. 49, 198-208.

Graf, D., R., H., Jones, C., M., Hallin, S. 2014. Intergenomic comparisons highlight modularity of the denitrification pathway and underpin the importance of community structure for $\mathrm{N}_{2} \mathrm{O}$ emissions. PLoS One. 9, e114118.

Gower, J.C. 1975. Generalized procrustes analysis. Psychmetrika. 40, 33-51.

Henry, S., Bru, D., Stres, B., Hallet, S., Philippot, L. 2006. Quantitative detection of the nos Z gene, encoding nitrous oxide reductase, and comparison of the abundances of $16 \mathrm{~S}$ rRNA, $n a r \mathrm{G}, \operatorname{nir} \mathrm{K}$, and $n o s \mathrm{Z}$ genes in soils. Appl. Environ. Microbiol. 72, 5181-5199.

Hube, S., Alfaro, M., Scheer, C., Brunk, C., Ramírez, L., Rowlings, D., Grace, P. 2016. Effect of a nitrification and urease inhibitors on nitrous oxide and methane emissions from an oat crop in a volcanic ash soil. Agric. Ecosyst. Environ. Accepted for publication. DOI http://dx.doi.org/10.1016/j.agee.2016.06.040
Jones, C.M., Graf, D.R.H., Bru, D., Philippot, L., Hallin, S. 2012. The unaccounted yet abundant nitrous oxide-reducing microbial community: a potential nitrous oxide sink. ISME J. 1-10.

Jorquera, M.A., Hernández, M., Martínez, O., Marschner, P., Mora, M. 2010. Detection of aluminium tolerance plasmids and microbial diversity in the rhizosphere of plants grown in acidic volcanic soil. Eur. J. Soil Biol. 46, 255263.

Kandeler, E., Deiglmayr, K., Tscherko, D., Bru, D., Philippot, L. 2006. Abundance of narG, nirS, $n i r \mathrm{~K}$, and $n o s \mathrm{Z}$ genes of denitrifying bacteria during primary successions of a glacier foreland. Appl. Environ. Microbiol. 72, 5957-5962.

Lagos, M.L., Maruyana, F., Nannipieri, P., Mora, M. L., Ogram, A., Jorquera, M.A. 2015. Current overview on the study of bacteria in the rhizosphere by modern molecular techniques: a mini-review. J. Soil Sci. Plant Nutr. 15, 504523.

Lobos Ortega, I., Alfaro, M., Martínez-Lagos, J. 2016. Soil nitrogen contribution to grasslands and its implication for nitrogen use efficiency. J. Soil Sci. Plant Nutr. 16(2).310-322.

Maeda, K., Spor, A., Edel-Hermann, V., Heraud, C., Breuil, M. C., Bizourd, F., Toyoda, S., Yoshida, N., Steinberg, C., Philippot, L. 2015. $\mathrm{N}_{2} \mathrm{O}$ production, a widespread trait in fungi. Sci. Rep. 5, 9697.

Michotey, V., Mejean, V., Bonin, P. 2000. Comparison of methods for quantification of cytochrome cd1-denitrifying bacteria in environmental Marine Samples. App. Environ. Microbiol. 66, 1564-1571.

Muñoz, C., Paulino, L., Vera, J., Zagal, E. 2011. $\mathrm{CO}_{2}$ and $\mathrm{N}_{2} \mathrm{O}$ emissions from an andisol in Chile under a NO-till system using non-fixed closed chambers. Chilean J. Agric. Res. 71, 275-282 
Muyzer, G., de Waal, E.C., Uitterlinden, A.G. 1993. Profiling of complex microbial populations by denaturing gradient gel electrophoresis analysis of polymerase chain reaction-amplified genes coding for 16S rRNA. Appl. Environ. Microbiol. 59, 695-700.

Nakatsu, C.H. 2007. Soil microbial community analysis using denaturing gradient gel electrophoresis. Soil Sci. Soc. Am. J. 71, 562.

Philippot, L., Hallin, S., Schloter, M. 2007. Ecology of denitrifying prokaryotes in agricultural soil. Advances in Agronomy. 96, 249-305.

Prévost-Bouré, N. C., Christen, R., Dequiedt, S., Mougel, C., Lelièvre, M., Jolivet, C., Shahbazkia, H. R., Guillou, L., Arrouays, D., Ranjard, L. 2011. Validation and application of a PCR primer set to quantify fungal communities in the soil environment by real-time quantitative PCR. PLoS One. 6, e24166.

Rowell, D.L. 1997. Soil science: Methods and Applications. Addison Wesley Longman Limited, Essex, pp: 79-108.

Rusch, A. 2013. Molecular Tools for the detection of nitrogen cycling Archaea. Archaea. 2013, 1-10.

Sadzawka, A., Carrasco, M.A., Demanet, R., Flores, H., Grez, R., Mora, M.L., Neaman, A. 2007. Métodos de análisis de tejidos vegetales. 2a ed. Serie Actas $\mathrm{N}^{\circ} 40$. Instituto de Investigaciones Agropecuarias INIA, Santiago, $139 \mathrm{p}$.
Sambrook, J., Rusell, D.W. 2001. Molecular cloning: A laboratory manual. 3rd ed. Cold Spring Harbor Laboratory Press, New York, NY, pp: 127-128.

Thomson, A. J., Giannopoulos, G., Pretty, J., Baggs, E.M., Richardson, D. 2012. Biological sources and sinks of nitrous oxide and strategies to mitigate emissions. Philos. Trans. R Soc. B Biol. Sci. $367,1157-1168$.

Throbäck, I.N., Enwall, K., Jarvis, A., Hallin, S. 2004. Reassessing PCR primers targeting nirS, nir $\mathrm{K}$ and nos $\mathrm{Z}$ genes for community surveys of denitrifying bacteria with DGGE. FEMS Microbiol. Ecol. 49, 401-417.

Vainio, E.J., Hantula, J. 2000. Direct analysis of wood-inhabiting fungi using denaturing gradient gel electrophoresis of amplified ribosomal DNA. Mycol. Res. 104, 927-936.

Vistoso, E., Alfaro, M., Saggar, S., Salazar, F. 2012. Effect of nitrogen inhibitors on nitrous oxide emissions and pasture growth after an autumn application in volcanic soil. Chil. J. Agric. Res. 72, 133-139.

Zar, J. H. 1999. Biostatistical Analysis. 4th Ed. Prentice Hall, New Jersey, 663 p. 